\title{
Normal integral bases and ray class groups
}

\author{
by \\ Humio Ichimura (Yokohama)
}

1. Introduction. A finite Galois extension $N / F$ over a number field $F$ with group $G$ has a normal integral basis (NIB for short) when $\mathcal{O}_{N}$ is cyclic over the group ring $\mathcal{O}_{F}[G]$. Here, $\mathcal{O}_{F}$ denotes the ring of integers of a number field $F$. For a prime number $p$, we say that a number field $F$ has property $\left(\mathrm{A}_{p}\right)$ when any tame cyclic extension $N / F$ of degree $p$ has a NIB. It is well known by Hilbert and Speiser that the rationals $\mathbb{Q}$ satisfy $\left(\mathrm{A}_{p}\right)$ for all primes $p$. On the other hand, Greither et al. [6] proved that any number field $F$ with $F \neq \mathbb{Q}$ does not satisfy $\left(\mathrm{A}_{p}\right)$ for some $p$. For an integer $a \in \mathcal{O}_{F}$, let $\mathrm{Cl}_{F}(a)$ be the ray ideal class group of $F$ defined modulo the principal ideal $a \mathcal{O}_{F}$. Using [6, Corollary 7], we showed in $[8, \mathrm{~V}]$ that if $\zeta_{p} \in F^{\times}$, then $F$ satisfies $\left(\mathrm{A}_{p}\right)$ if and only if the ray class group $\mathrm{Cl}_{F}(p)$ is trivial. Here, $\zeta_{p}$ is a primitive $p$ th root of unity.

Let $p \geq 3$ be a prime number, $F$ a number field, $K=F\left(\zeta_{p}\right)$, and $\Delta_{F}=\operatorname{Gal}(K / F)$. In this paper, we study property $\left(\mathrm{A}_{p}\right)$ when $\zeta_{p} \notin F^{\times}$but $[K: F]=2$ in connection with the ray class groups $\mathrm{Cl}_{K}(\pi)$ and $\mathrm{Cl}_{K}(p)$. Here, $\pi=\pi_{p}=\zeta_{p}-1$. For a set $X$ on which $\Delta_{F}$ acts, $X^{\Delta_{F}}$ denotes the invariant part. First, we prove the following:

THEOREM 1. Let $p \geq 3$ be a prime number, $F$ a number field such that $\zeta_{p} \notin F^{\times}$, and $K=F\left(\zeta_{p}\right)$. Assume that $[K: F]=2$. If $F$ satisfies $\left(\mathrm{A}_{p}\right)$, then the ray class groups $\mathrm{Cl}_{K}(\pi)$ and $\mathrm{Cl}_{K}(p)^{\Delta_{F}}$ are trivial.

We say that a number field $F$ has property $\left(\mathrm{B}_{p}\right)$ when for any $a \in F^{\times}$, the cyclic extension $K\left(a^{1 / p}\right) / K$ has a NIB if it is tame, where $K=F\left(\zeta_{p}\right)$. A theorem of Kawamoto $[12,13]$ asserts that $\mathbb{Q}$ satisfies $\left(\mathrm{B}_{p}\right)$ for all primes $p$. Analogously to the result of Greither et al., it is known by $[8, \mathrm{IV}]$ that any number field $F \neq \mathbb{Q}$ does not satisfy $\left(\mathrm{B}_{p}\right)$ for some $p$. In [11], we studied this property in some more detail. Using Theorem 1 , we prove the following "duality" between properties $\left(\mathrm{A}_{p}\right)$ and $\left(\mathrm{B}_{p}\right)$.

2000 Mathematics Subject Classification: Primary 11R33. 
THEOREM 2. Under the setting and assumptions of Theorem 1, assume further that $K / F$ is ramified at least for one prime divisor (including the infinite one). Then $F$ satisfies $\left(\mathrm{A}_{p}\right)$ only when it satisfies $\left(\mathrm{B}_{p}\right)$.

When $p=3$, we can prove the following assertion stronger than Theorem 1.

Theorem 3. Let $p=3, F$ be a number field with $\zeta_{3} \notin F^{\times}$, and $K=$ $F\left(\zeta_{3}\right)$. Then $F$ satisfies $\left(\mathrm{A}_{3}\right)$ if and only if the ray class groups $\mathrm{Cl}_{K}\left(\pi_{3}\right)$ and $\mathrm{Cl}_{K}(3)^{\Delta_{F}}$ are trivial.

To prove this, we need the following descent property on NIB.

Theorem 4. Let $p=3$, and $F, K$ be as in Theorem 3. Then a tame cyclic cubic extension $N / F$ has a NIB if and only if $N K / K$ has a NIB.

When $p=3$ is unramified at $F$, this assertion is already known, by a result of Greither [5, Theorem 2.2].

The following is a consequence of Theorem 3 .

Proposition. Let $p=3$ and $F=\mathbb{Q}(\sqrt{d})$ be a quadratic field with $d a$ square free integer. Then $F$ satisfies $\left(\mathrm{A}_{3}\right)$ if and only if

$$
d=-1,-2,-3,-11,2,3,5,6,17,33,41,89 .
$$

Remark 1. Let $p=2$ and $K$ be a number field. It is shown in $[8, \mathrm{~V}]$ that the following three conditions are equivalent:

(i) any tame abelian extension over $K$ of exponent 2 has a NIB,

(ii) any tame $(2,2)$-extension over $K$ has a NIB,

(iii) the ray class group $\mathrm{Cl}_{K}$ (4) of $K$ defined modulo 4 is trivial.

REMARK 2. Let $h_{p}$ (resp. $h_{p}^{+}$) be the class number of the $p$-cyclotomic field $\mathbb{Q}\left(\zeta_{p}\right)$ (resp. $\left.\mathbb{Q}(\cos (2 \pi / p))\right)$, and let $h_{p}^{-}=h_{p} / h_{p}^{+}$. It is well known by Kummer that if $p \nmid h_{p}^{-}$, then $p \nmid h_{p}^{+}$(cf. Washington [15, Theorems 5.34, 10.9]). Under the setting of Theorem 1 , let $X$ be the subgroup of $K^{\times} /\left(K^{\times}\right)^{p}$ consisting of classes $[x]$ with $x \in K^{\times}$such that the cyclic extension $K\left(x^{1 / p}\right) / K$ is tame. Namely,

$$
X=\left\{[x] \in K^{\times} /\left(K^{\times}\right)^{p} \mid(x, p)=1, x \equiv u^{p} \bmod \pi^{p} \text { for some } u \in \mathcal{O}_{K}\right\} .
$$

By the action of $\Delta_{F}$, we can decompose $X$ as $X=X^{+} \oplus X^{-}$, where $X^{+}$ $=X^{\Delta_{F}}$. Property $\left(\mathrm{A}_{p}\right)\left(\operatorname{resp} .\left(\mathrm{B}_{p}\right)\right)$ is a property on $X^{-}\left(\operatorname{resp} . X^{+}\right)$. Hence, Theorem 2 may be regarded as a Galois module analogue of the above classical duality.

REMARK 3. In [11, Theorem 2], we proved that an imaginary quadratic field $F=\mathbb{Q}(\sqrt{d})$ with $d$ a square free negative integer satisfies $\left(\mathrm{B}_{3}\right)$ if and only if $d=-1,-2,-3$, or -11 . Hence, by the Proposition, $\left(\mathrm{A}_{3}\right)$ and $\left(\mathrm{B}_{3}\right)$ are equivalent for imaginary quadratic fields. However, in general, $\left(\mathrm{A}_{p}\right)$ is 
stronger than $\left(\mathrm{B}_{p}\right)$. Actually, we see from [11, Theorem 3] and the Proposition that there are many real quadratic fields satisfying $\left(\mathrm{B}_{3}\right)$ but not $\left(\mathrm{A}_{3}\right)$.

REMARK 4. On the descent property on NIB, the following general fact is known for the unramified case. Let $p \geq 3$ be a prime number, $F$ a number field with $\zeta_{p} \notin F^{\times}$, and $K=F\left(\zeta_{p}\right)$. Then an unramified cyclic extension $N / F$ of degree $p$ has a NIB if and only if $N K / K$ has a NIB. This was first shown by Brinkhuis [1] when $p=3$ and $F$ is an imaginary quadratic field, and then by the author [9] for the general case.

This paper is organized as follows. In Section 2, we recall a theorem of Gómez Ayala on NIB, and give some of its versions. In Section 3, we show several lemmas related to the theorem. In Section 4, we prove Theorems 1 and 2. In Section 5, we deal with the case $p=3$, and prove Theorems 3, 4 and the Proposition.

2. A theorem of Gómez Ayala. In this section, we recall a theorem of Gómez Ayala on NIB and give some of its versions. Let $p$ be a prime number, $K$ a number field with $\zeta_{p} \in K^{\times}, L / K$ a cyclic extension of degree $p$, and $G=\operatorname{Gal}(L / K)$. Let $g$ be a fixed generator of $G$ and $\zeta_{p}$ a fixed primitive $p$ th root of unity. For $0 \leq i \leq p-1$, let $\mathcal{O}_{L}^{(i)}$ be the additive group of integers $x \in \mathcal{O}_{L}$ such that $x^{g}=\zeta_{p}^{i} x$. For an integer $\omega \in \mathcal{O}_{L}$, the resolvent $\omega_{i}$ is defined by

$$
\omega_{i}=\sum_{r=0}^{p-1} \zeta_{p}^{-i r} \omega^{g^{r}} \quad(0 \leq i \leq p-1) .
$$

As is easily seen, we have $\omega_{i} \in \mathcal{O}_{L}^{(i)}$. The following lemma is easily shown and well known to specialists. Let $E_{K}=\mathcal{O}_{K}^{\times}$be the group of units of $K$.

LEMMA 1. Under the above setting, the following assertions hold. If $L / K$ has a NIB, then $\mathcal{O}_{L}^{(i)}$ is cyclic over $\mathcal{O}_{K}$ for each $i$. More precisely, if an integer $\omega$ of $L$ generates $\mathcal{O}_{L}$ over $\mathcal{O}_{K}[G]$, then the resolvent $\omega_{i}$ generates $\mathcal{O}_{L}^{(i)}$ over $\mathcal{O}_{K}$ and

$$
\omega_{0} \in E_{K} \quad \text { and } \quad \sum_{i=0}^{p-1} \omega_{i} \equiv 0 \bmod p .
$$

In [4, Theorem 2.1], Gómez Ayala gave the following necessary and sufficient condition for a tame Kummer extension of prime degree to have a NIB in terms of a Kummer generator. Let $\mathfrak{A}$ be a $p$ th power free integral ideal of a number field $K$. Namely, $\wp^{p} \nmid \mathfrak{A}$ for any prime ideal $\wp$ of $K$. Then we can uniquely write

$$
\mathfrak{A}=\prod_{i=1}^{p-1} \mathfrak{A}_{i}^{i}
$$


for some square free integral ideals $\mathfrak{A}_{i}$ of $K$ relatively prime to each other. The associated ideals $\mathfrak{B}_{j}$ of $\mathfrak{A}$ are defined by

$$
\mathfrak{B}_{j}=\prod_{i=1}^{p-1} \mathfrak{A}_{i}^{[i j / p]} \quad(0 \leq j \leq p-1) .
$$

Here, for a real number $x,[x]$ denotes the largest integer $\leq x$. Clearly, we have $\mathfrak{B}_{0}=\mathfrak{B}_{1}=\mathcal{O}_{K}$.

Theorem 5 (Gómez Ayala). Let $p$ be a prime number and $K$ a number field with $\zeta_{p} \in K^{\times}$. Let $L / K$ be a tame cyclic extension of degree $p$ with group $G$. Then $L / K$ has a NIB if and only if there exists an integer a $\in \mathcal{O}_{L}$ with $L=K\left(a^{1 / p}\right)$ satisfying the following three conditions:

(i) the integral ideal $a \mathcal{O}_{K}$ is pth power free,

(ii) the associated ideals $\mathfrak{B}_{j}$ of $a \mathcal{O}_{K}$ defined by (1) and (2) are principal,

(iii) letting $\alpha=a^{1 / p}$, the congruence

$$
A=\sum_{j=0}^{p-1} \frac{\alpha^{j}}{x_{j}} \equiv 0 \bmod p
$$

holds for some generators $x_{j}$ of the principal ideals $\mathfrak{B}_{j}$.

Further, when this is the case, $\omega=A / p$ generates $\mathcal{O}_{L}$ over $\mathcal{O}_{K}[G]$.

The following is a consequence of this theorem.

Lemma 2. Let $p, K, L / K, G$ be as in Theorem 5 . Then $L / K$ has a NIB if and only if the following conditions are satisfied:

(i) $\mathcal{O}_{L}^{(i)}$ is cyclic over $\mathcal{O}_{K}$ for each $i$ with $0 \leq i \leq p-1$,

(ii) there exists a generator $\alpha_{i}$ of $\mathcal{O}_{L}^{(i)}$ over $\mathcal{O}_{K}$ such that the principal ideal $\alpha_{i}^{p} \mathcal{O}_{K}$ of $K$ is pth power free and

$$
A=\sum_{i=0}^{p-1} \alpha_{i} \equiv 0 \bmod p .
$$

Further, when this is the case, $\omega=A / p$ generates $\mathcal{O}_{L}$ over $\mathcal{O}_{K}[G]$.

Proof. Assume that $L / K$ has a NIB. Under the notation of Theorem 5 , let $g$ be a generator of $G$ with $\alpha^{g}=\zeta_{p} \alpha$. By Lemma $1, \mathcal{O}_{L}^{(i)}$ is cyclic over $\mathcal{O}_{K}$. Further, we see from (1) and (2) that $\alpha^{i} / x_{i} \in \mathcal{O}_{L}^{(i)}$ and $\left(a^{i} / x_{i}^{p}\right) \mathcal{O}_{K}$ is $p$ th power free. Therefore, $\alpha_{i}=\alpha^{i} / x_{i}$ generates $\mathcal{O}_{L}^{(i)}$ over $\mathcal{O}_{K}$. Hence, conditions (i) and (ii) of Lemma 2 are satisfied by Theorem 5 . Conversely, assume that these two conditions are satisfied. Let $\alpha=\alpha_{1}$ and $a=\alpha^{p}\left(\in \mathcal{O}_{K}\right)$, and choose $g \in G$ so that $\alpha^{g}=\zeta_{p} \alpha$. Clearly, $L=K\left(a^{1 / p}\right)$, and condition (i) in Theorem 5 is satisfied. Let $\mathfrak{B}_{j}$ be the ideals associated to the $p$ th power free 
integral ideal $a \mathcal{O}_{K}$. We have $\alpha_{j}=\alpha^{j} / y_{j}$ for some $y_{j} \in \mathcal{O}_{K}$ since $\alpha^{j} \in \mathcal{O}_{L}^{(j)}$ and $\alpha_{j}$ generates $\mathcal{O}_{L}^{(j)}$ over $\mathcal{O}_{K}$. However, as $\alpha_{j}^{p} \mathcal{O}_{K}$ is $p$ th power free, we see that $y_{j} \mathcal{O}_{K}=\mathfrak{B}_{j}$ by (2). Therefore, all conditions in Theorem 5 are satisfied, and hence $L / K$ has a NIB.

The following is a version of this lemma.

Lemma 3. Let $p \geq 3$ be a prime number, $F$ a number field with $\zeta_{p} \notin F^{\times}$, and $K=F\left(\zeta_{p}\right)$. Assume that $[K: F]=2$. Let $N / F$ be a tame cyclic extension of degree $p$ with group $G$, and $L=N K$. Then $N / F$ has a NIB if and only if the following two conditions are satisfied:

(i) $\mathcal{O}_{L}^{(i)}$ is cyclic over $\mathcal{O}_{K}$ for each $i$ with $0 \leq i \leq(p-1) / 2$,

(ii) for each $0 \leq i \leq(p-1) / 2$, there exists a generator $\alpha_{i}$ of $\mathcal{O}_{L}^{(i)}$ over $\mathcal{O}_{K}$ such that the principal ideal $\alpha_{i}^{p} \mathcal{O}_{K}$ of $K$ is pth power free and

$$
\alpha_{0} \in E_{F} \quad \text { and } \quad A=\alpha_{0}+\sum_{i=1}^{(p-1) / 2}\left(\alpha_{i}+\alpha_{i}^{\prime}\right) \equiv 0 \bmod p .
$$

Here and in the proof of this lemma, for an element $x$ (resp. a subset $X$ ) of $L, x^{\prime}$ (resp. $X^{\prime}$ ) denotes its conjugate over $N$.

Proof. Since $L=N K$ and $\zeta_{p}^{\prime}=\zeta_{p}^{-1}$, we easily see that

$$
\left(\mathcal{O}_{L}^{(i)}\right)^{\prime}=\mathcal{O}_{L}^{(p-i)}, \quad \omega_{i}^{\prime}=\omega_{p-i} \quad(1 \leq i \leq(p-1) / 2)
$$

for any integer $\omega \in \mathcal{O}_{N}$. Assume that $\mathcal{O}_{N}=\mathcal{O}_{F}[G] \omega$ for some $\omega \in \mathcal{O}_{N}$. Then we see that $\mathcal{O}_{L}=\mathcal{O}_{K}[G] \omega$ by a classical result on rings of integers (cf. Fröhlich and Taylor [3, III, (2.13)]), and that

$$
\omega_{0}=\operatorname{Tr}_{L / K} \omega \in E_{K} \cap F=E_{F}
$$

by Lemma 1. Here, $\operatorname{Tr}_{L / K}$ denotes the trace map. From the above and Lemmas 1, 2, we see that conditions (i) and (ii) of Lemma 3 are satisfied with $\alpha_{i}=\omega_{i}$. Conversely, assume that conditions (i) and (ii) are satisfied, and let $\omega=A / p$. Then $\omega \in \mathcal{O}_{N}$ by (ii). By (3) and the conditions of Lemma 3, we see from Lemma 2 that $\mathcal{O}_{L}=\mathcal{O}_{K}[G] \omega$. As $\omega \in \mathcal{O}_{N}$, this implies that $\mathcal{O}_{N}=\mathcal{O}_{F}[G] \omega$.

REMARK 5. In [10], we gave a generalization of the theorem of Gómez Ayala (Theorem 5) and some of its applications. A function field analogue of the theorem is already given in Chapman [2].

3. Lemmas. In this section, we prepare some lemmas related to Theorem 5 which are necessary to prove our theorems. In what follows, we let $p \geq 3$ be a fixed odd prime number, $\zeta_{p}$ a fixed primitive $p$ th root of unity, and $\pi=\pi_{p}=\zeta_{p}-1$. 
Lemma 4. Let $s, t$ be integers with $1 \leq s<t \leq p-1$. Let $K$ be a number field, and $\mathfrak{A}_{1}, \mathfrak{A}_{2}$ square free integral ideals of $K$ relatively prime to each other. If the associated ideals $\mathfrak{B}_{j}$ of $\mathfrak{A}=\mathfrak{A}_{1}^{s} \mathfrak{A}_{2}^{t}$ defined by (2) are principal, then $\mathfrak{A}_{1}$ and $\mathfrak{A}_{2}$ are principal.

Proof. As $s<t$, we see that $[s i / p]<[t i / p]$ for some $i$ with $1 \leq i \leq p-1$. Let $k$ be the smallest such integer. Then

$$
[s(k-1) / p]=[t(k-1) / p]=[s k / p]=a, \quad[t k / p]=a+1
$$

for some integer $a$. This is because $[s(i+1) / p]=[s i / p]$ or $[s i / p]+1$. Therefore, from the assumption, the ideals $\mathfrak{B}_{k-1}=\mathfrak{A}_{1}^{a} \mathfrak{A}_{2}^{a}$ and $\mathfrak{B}_{k}=\mathfrak{A}_{1}^{a} \mathfrak{A}_{2}^{a+1}$ are principal. Hence, $\mathfrak{A}_{2}$ is principal. Let $r$ be the smallest integer with $[s r / p] \geq 1$. Then $[s r / p]=1$ and $\mathfrak{B}_{r}=\mathfrak{A}_{1} \mathfrak{A}_{2}^{[t r / p]}$. Hence, $\mathfrak{A}_{1}$ is also principal.

Lemma 5. Let $K$ be a number field with $\zeta_{p} \in K^{\times}$, and let $\lambda_{1}$ and $\lambda_{2}$ be integers of $K$ such that the principal ideals $\lambda_{1} \mathcal{O}_{K}$ and $\lambda_{2} \mathcal{O}_{K}$ are square free and relatively prime to each other and to $p$. Let $a=\lambda_{1} \lambda_{2}^{p-1}$, and $L=$ $K\left(a^{1 / p}\right)$. Assume that $L / K$ has a NIB. Then $a \equiv \eta^{p} \bmod \pi^{p}$ and $\lambda_{i} \equiv$ $\eta_{i} \bmod \pi$ for some units $\eta, \eta_{i} \in E_{K}$ with $i=1$, 2 . Further, when $p=3$, we have $\lambda_{i} \equiv \eta_{i} \bmod 3$ for some $\eta_{i} \in E_{K}$.

Proof. When $a \in\left(K^{\times}\right)^{p}$, we easily see that $a, \lambda_{1}$ and $\lambda_{2}$ are units of $K$ from the conditions on $\lambda_{i}$, and hence the assertion is obvious. So, we may as well assume that $[L: K]=p$. Let $\alpha=a^{1 / p}$, and choose a generator $g$ of $\operatorname{Gal}(L / K)$ so that $\alpha^{g}=\zeta_{p} \alpha$. For $1 \leq i \leq p-1$, let $\alpha_{i}=\alpha^{i} / \lambda_{2}^{i-1}$. Then $\alpha_{i} \in \mathcal{O}_{L}^{(i)}$, and the ideal $\alpha_{i}^{p} \mathcal{O}_{K}=\lambda_{1}^{i} \lambda_{2}^{p-i} \mathcal{O}_{K}$ of $K$ is $p$ th power free. Hence, by the assumption and Lemma 1, we have $\mathcal{O}_{L}^{(i)}=\mathcal{O}_{K} \alpha_{i}$. Therefore, by Lemma 2, the congruence

$$
\delta_{0}+\delta_{1} \alpha+\delta_{2} \frac{\alpha^{2}}{\lambda_{2}}+\ldots+\delta_{p-1} \frac{\alpha^{p-1}}{\lambda_{2}^{p-2}} \equiv 0 \bmod p
$$

holds for some units $\delta_{i} \in E_{K}$. It follows from this that

$$
\sum_{i=1}^{p-1} \delta_{p-i} \lambda_{2}^{i-1} \alpha^{p-i}+\delta_{0} \lambda_{2}^{p-2} \equiv 0 \bmod p,
$$

and that

$$
\frac{\delta_{0}}{\alpha^{2}}+\frac{\delta_{1}}{\alpha}+\frac{\delta_{2}}{\lambda_{2}}+\sum_{i=1}^{p-3} \frac{\delta_{i+2}}{\lambda_{2}^{i+1}} \alpha^{i} \equiv 0 \bmod p .
$$

For a congruence $(*)$ such as $(4)$ and $(5)$, let $(*)^{g}$ be the congruence obtained by letting $g$ act on $(*)$. Let

$$
c_{j}=1+\zeta_{p}+\zeta_{p}^{2}+\ldots+\zeta_{p}^{j}=\frac{\zeta_{p}^{j+1}-1}{\zeta_{p}-1} \quad(0 \leq j \leq p-2)
$$


be a cyclotomic unit. Dividing $(4)-(4)^{g}$ by $\pi \alpha$, we obtain

$$
\sum_{i=1}^{p-2} \delta_{p-i} c_{p-1-i} \lambda_{2}^{i-1} \alpha^{p-1-i}+\delta_{1} \lambda_{2}^{p-2} \equiv 0 \bmod \pi^{p-2} .
$$

Again, dividing $(6)-(6)^{g}$ by $\pi \alpha$, we obtain

$$
\sum_{i=1}^{p-3} \delta_{p-i} c_{p-1-i} c_{p-2-i} \lambda_{2}^{i-1} \alpha^{p-2-i}+\delta_{2} c_{1} \lambda_{2}^{p-3} \equiv 0 \bmod \pi^{p-3}
$$

Repeating this process, we finally obtain

$$
\delta_{p-1}\left(\prod_{i=1}^{p-2} c_{i}\right) \alpha+\delta_{p-2}\left(\prod_{i=0}^{p-3} c_{i}\right) \lambda_{2} \equiv 0(\bmod \pi)
$$

and hence

$$
\alpha \equiv \frac{\delta_{p-2}}{\delta_{p-1}} \lambda_{2} \bmod \pi
$$

Starting from the congruence (5), we similarly obtain

$$
\alpha \equiv \delta_{0} / \delta_{1} \bmod \pi \text {. }
$$

From the last two congruences, we obtain the assertion for the general case.

Finally, let us deal with the case $p=3$. By (4), we have

$$
\delta_{2} \alpha^{2}+\delta_{1} \lambda_{2} \alpha+\delta_{0} \lambda_{2} \equiv 0 \bmod 3 \text {. }
$$

For an element $x \in L^{\times}$with $x \equiv 1 \bmod \pi_{3}$, we have $x^{2}+x+1 \equiv 0 \bmod 3$. Hence, it follows from (7) that

$$
\delta_{2} \alpha^{2}+\delta_{1} \lambda_{2} \alpha+\delta_{1}^{2} \delta_{2}^{-1} \lambda_{2}^{2} \equiv 0 \bmod 3 .
$$

From these two congruences, it follows that $\lambda_{2} \equiv \delta_{0} \delta_{1}^{-2} \delta_{2} \bmod 3$. The assertion for the case $p=3$ follows from this.

For an ideal $\mathfrak{A}$ of $K$ with $(\mathfrak{A}, p)=1$, let $[\mathfrak{A}]_{\pi}$ be the ideal class in $\mathrm{Cl}_{K}(\pi)$ represented by $\mathfrak{A}$.

LEMMA 6. Let $K$ be a number field with $\zeta_{p} \in K^{\times}, a \in \mathcal{O}_{K}$ an integer such that $a \equiv 1 \bmod \pi^{p}$, and $L=K\left(a^{1 / p}\right)$. Assume that (i) $L / K$ has a $N I B$, and (ii) $a \mathcal{O}_{K}=\mathfrak{A}_{1}^{r} \mathfrak{A}_{2}^{p-r} \mathfrak{A}_{3}^{p}$ for some $1 \leq r \leq p-1$ and some integral ideals $\mathfrak{A}_{i}$ of $K$ such that $\mathfrak{A}_{1}$ and $\mathfrak{A}_{2}$ are square free and relatively prime to each other. Then the classes $\left[\mathfrak{A}_{i}\right]_{\pi} \in \mathrm{Cl}_{K}(\pi)$ are trivial for $i=1,2,3$.

Proof. As $L / K$ has a NIB, we see from Theorem 5 that there exists an integer $b \in \mathcal{O}_{K}$ with $L=K\left(b^{1 / p}\right)$ such that $b \mathcal{O}_{K}$ is $p$ th power free and the ideals associated to $b \mathcal{O}_{K}$ by (1) and (2) are principal. By assumption (ii), we see that $b \mathcal{O}_{K}=\mathfrak{A}_{1}^{s} \mathfrak{A}_{2}^{p-s}$ for some $1 \leq s \leq p-1$. It follows from Lemma 4 that $\mathfrak{A}_{i}=\lambda_{i} \mathcal{O}_{K}$ for some $\lambda_{i} \in \mathcal{O}_{K}$ with $i=1,2$. Then

$$
L=K\left(\left(\varepsilon_{1} \lambda_{1} \lambda_{2}^{p-1}\right)^{1 / p}\right)
$$


for some unit $\varepsilon_{1} \in E_{K}$. As $L / K$ has a NIB, we see from Lemma 5 that $\lambda_{i} \equiv \eta_{i} \bmod \pi$. Hence, the classes $\left[\mathfrak{A}_{1}\right]_{\pi}$ and $\left[\mathfrak{A}_{2}\right]_{\pi}$ are trivial. It also follows from Lemma 5 that $\varepsilon_{1} \lambda_{1} \lambda_{2}^{p-1} \equiv \eta^{p} \bmod \pi^{p}$ for some $\eta \in E_{K}$. From this and $\lambda_{2} \equiv \eta_{2} \bmod \pi$, it follows that

$$
\varepsilon_{1}^{r} \lambda_{1}^{r} \lambda_{2}^{p-r} \equiv \delta^{p} \bmod \pi^{p}
$$

for some $\delta \in E_{K}$. From assumption (ii) and (8), we see that $a=\varepsilon_{1}^{r} \lambda_{1}^{r} \lambda_{2}^{p-r} x^{p}$ for some $x \in \mathcal{O}_{K}$ and that $\mathfrak{A}_{3}=x \mathcal{O}_{K}$. Then, by (9) and $a \equiv 1 \bmod \pi^{p}$, it follows that $x$ is congruent to a unit modulo $\pi$. Therefore, $\left[\mathfrak{A}_{3}\right]_{\pi}=1$.

For a number field $K$ and an integer $a \in \mathcal{O}_{K}$, we write $\mathcal{O}_{K} / a=\mathcal{O}_{K} / a \mathcal{O}_{K}$ for brevity, and let $\left[E_{K}\right]_{a}$ be the subgroup of the multiplicative group $\left(\mathcal{O}_{K} / a\right)^{\times}$generated by the classes containing units of $K$. For an element $x \in K^{\times}$with $(x, a)=1,[x]_{a}$ denotes the class represented by $x$. When $F$ is a subfield of $K$ and $a \in \mathcal{O}_{F}$, we naturally regard $\left(\mathcal{O}_{F} / a\right)^{\times}$as a subgroup of $\left(\mathcal{O}_{K} / a\right)^{\times}$.

Lemma 7. (I) For a number field $K$ with $\zeta_{p} \in K^{\times}$, the exponent of $\mathrm{Cl}_{K}(p)$ divides $p$ if $\mathrm{Cl}_{K}(\pi)=\{0\}$.

(II) Let $F$ be a number field with $\zeta_{p} \notin F^{\times}$, and $K=F\left(\zeta_{p}\right)$. Assume that $\mathrm{Cl}_{K}(\pi)=\{0\}$. Then $\mathrm{Cl}_{K}(p)^{\Delta_{F}}=\{0\}$ if and only if $\left(\mathcal{O}_{F} / p\right)^{\times} \subseteq\left[E_{K}\right]_{p}$.

Proof. We put

$$
A=\left(1+\pi \mathcal{O}_{K}\right) /\left(1+p \mathcal{O}_{K}\right)\left(\subseteq\left(\mathcal{O}_{K} / p\right)^{\times}\right) \text {and } B=A\left[E_{K}\right]_{p} /\left[E_{K}\right]_{p} .
$$

As $\mathrm{Cl}_{K}(\pi)=\{0\}$, we see that $\mathrm{Cl}_{K}(p)=B$ from the exact sequence

$$
\{0\} \rightarrow B \rightarrow \mathrm{Cl}_{K}(p) \rightarrow \mathrm{Cl}_{K}(\pi) \rightarrow\{0\} .
$$

Then the first assertion is obvious as $A$ is of exponent $p$. Let us show the second one. The "only if" part holds as $\left(\mathcal{O}_{K} / p\right)^{\times} /\left[E_{K}\right]_{p}$ is a subgroup of $\mathrm{Cl}_{K}(p)$. We show the "if" part. We have $\mathrm{Cl}_{K}(p)=B$ as $\mathrm{Cl}_{K}(\pi)=\{0\}$. Let $d=[K: F]$, and let $\mathcal{C}=[x]_{p}$ be a class in $B^{\Delta_{F}}$ with $x \in \mathcal{O}_{K}$. Then, since $\mathcal{C}^{d}=\left[N_{K / F} x\right]_{p}$, we obtain $\mathcal{C}^{d}=1$ by $\left(\mathcal{O}_{F} / p\right)^{\times} \subseteq\left[E_{K}\right]_{p}$. Here, $N_{K / F}$ denotes the norm map. Therefore, $\mathcal{C}=1$ as $d$ divides $p-1$ and $B$ is a $p$-abelian group.

Let $F$ be a number field with $\zeta_{p} \notin F^{\times}$, and $K=F\left(\zeta_{p}\right)$. When $[K: F]$ $=2$, for an element $x$ (resp. an ideal $\mathfrak{A})$ of $K$, let $x^{\prime}$ (resp. $\mathfrak{A}^{\prime}$ ) denotes the conjugate over $F$.

Lemma 8. Let $F$ be a number field with $\zeta_{p} \notin F^{\times}$, and $K=F\left(\zeta_{p}\right)$. Assume that $[K: F]=2$. Assume further that $\mathrm{Cl}_{K}(\pi)$ and $\mathrm{Cl}_{K}(p)^{\Delta_{F}}$ are trivial. Let $a$ be an integer of $K$ relatively prime to $p$ such that $a \mathcal{O}_{K}$ is square free and $\left(a, a^{\prime}\right)=1$, and let $b=a\left(a^{\prime}\right)^{p-1}$. Then the cyclic extension $L=K\left(b^{1 / p}\right) / K$ has a NIB if it is tame. 
Proof. Let $\mathfrak{B}_{j}$ be the ideals of $K$ associated to $b \mathcal{O}_{K}$ by (1) and (2). Then

$$
\mathfrak{B}_{0}=\mathfrak{B}_{1}=\mathcal{O}_{K}, \quad \mathfrak{B}_{j}=\left(a^{\prime}\right)^{j-1} \mathcal{O}_{K} \quad \text { for } 2 \leq j \leq p-1 .
$$

As $L / K$ is tame, $b \equiv u^{p} \bmod \pi^{p}$ for some $u \in \mathcal{O}_{K}$. We see that $u \equiv \varepsilon \bmod \pi$ for some unit $\varepsilon \in E_{K}$ because $\mathrm{Cl}_{K}(\pi)=\{0\}$. Hence, $b=a\left(a^{\prime}\right)^{p-1} \equiv$ $\varepsilon^{p} \bmod \pi^{p}$. On the other hand, $a a^{\prime}\left(\in \mathcal{O}_{F}\right)$ is congruent to a unit modulo $p$ since $\mathrm{Cl}_{K}(p)^{\Delta_{F}}=\{0\}$. Hence, so is $\left(a^{\prime}\right)^{p-2}=b /\left(a a^{\prime}\right)$. This implies that $a^{\prime} \equiv \delta \bmod p$ for some unit $\delta \in E_{K}$ because $\mathrm{Cl}_{K}(p)$ and its subgroup $\left(\mathcal{O}_{K} / p\right)^{\times} /\left[E_{K}\right]_{p}$ are $p$-abelian groups (by Lemma 7 ). Now, let $\beta=b^{1 / p}$ $(\equiv \varepsilon \bmod \pi)$. Then

$$
1+\frac{\beta}{\varepsilon}+\sum_{j=2}^{p-1} \delta^{j-1} \frac{\beta^{j}}{\left(a^{\prime}\right)^{j-1} \varepsilon^{j}} \equiv \sum_{j=0}^{p-1}(\beta / \varepsilon)^{j} \equiv 0 \bmod p .
$$

Therefore, $L / K$ has a NIB by Theorem 5 .

The following is one more consequence of Theorem 5, for which see [11, Proposition 1]. For a number field $F$, let $h_{F}$ be the class number of $F$ (in the usual sense).

Lemma 9. Let $p$ be a prime number, $F$ a number field, and $K=F\left(\zeta_{p}\right)$. Assume that $h_{F}=1$ and $\left(\mathcal{O}_{F} / p\right)^{\times} \subseteq\left[E_{K}\right]_{p}$. Then $F$ satisfies $\left(\mathrm{B}_{p}\right)$.

4. Proofs of Theorems 1 and 2. First, we derive Theorem 2 from Theorem 1.

Proof of Theorem 2. Assume that $F$ satisfies $\left(\mathrm{A}_{p}\right)$. Then $\mathrm{Cl}_{K}(\pi)$ and $\mathrm{Cl}_{K}(p)^{\Delta_{F}}$ are trivial by Theorem 1 . Since $[K: F]=2$ and $\mathrm{Cl}_{K}(p)^{\Delta_{F}}=\{0\}$, we see that $h_{F}=1$, or $h_{F}=2$ and $K / F$ is the Hilbert class field of $F$. Hence, by the second assumption of Theorem 2, we must have $h_{F}=1$. On the other hand, $\left(\mathcal{O}_{F} / p\right)^{\times} \subseteq\left[E_{K}\right]_{p}$ by Lemma 7 . Hence, $F$ satisfies $\left(\mathrm{B}_{p}\right)$ by Lemma 9.

To prove Theorem 1, the following theorem of Greither et al. [6, Corollary] is crucial.

THEOREM 6 (Greither et al.). If a number field $F$ has property $\left(\mathrm{A}_{p}\right)$, then the exponent of the quotient $\left(\mathcal{O}_{F} / p\right)^{\times} /\left[E_{F}\right]_{p}$ divides $(p-1)^{2} / 2$.

Proof of Theorem 1. This is done in several steps.

Lemma 10. Under the setting of Theorem 1 , assume that $[K: F]=2$ and that $F$ satisfies $\left(\mathrm{A}_{p}\right)$. Then $\mathrm{Cl}_{K}\left(\pi^{p}\right)$ is a p-abelian group. Hence, $\mathrm{Cl}_{K}(p)$, $\mathrm{Cl}_{K}(\pi)$ and $\mathrm{Cl}_{K}$ are $p$-abelian groups.

Proof. Let $\mathcal{C}$ be an ideal class in $\mathrm{Cl}_{K}\left(\pi^{p}\right)$ whose order $n$ is relatively prime to $p$. It suffices to show that $\mathcal{C}=1$. Let $\mathfrak{P} \in \mathcal{C}$ be a prime ideal 
of $K$ with $\left(\mathfrak{P}, \mathfrak{P}^{\prime}\right)=1$. Then $\mathfrak{P}^{n}=a \mathcal{O}_{K}$ for some integer $a \in \mathcal{O}_{K}$ with $a \equiv 1 \bmod \pi^{p}$. Let $b=a\left(a^{\prime}\right)^{p-1}$ and $L=K\left(b^{1 / p}\right)$. Since

$$
b \mathcal{O}_{K}=\mathfrak{P}^{n}\left(\mathfrak{P}^{\prime}\right)^{n(p-1)} \text { and } p \nmid n,
$$

the extension $L / K$ is of degree $p$. It is tame as $b \equiv 1 \bmod \pi^{p}$. As $b b^{\prime} \in\left(K^{\times}\right)^{p}$, there uniquely exists a tame cyclic extension $N / F$ of degree $p$ with $L=N K$. As $F$ satisfies $\left(\mathrm{A}_{p}\right), N / F$ and hence $L / K$ have a NIB. Now, it follows from (10) and Lemma 6 that $\mathfrak{P}=\lambda \mathcal{O}_{K}$ for some $\lambda \in \mathcal{O}_{K}$ and that $\lambda \equiv \delta \bmod \pi$ for some $\delta \in E_{K}$. As $\lambda^{p} \equiv \delta^{p} \bmod \pi^{p}$, we see that $\mathcal{C}^{p}$ is trivial in $\mathrm{Cl}_{K}\left(\pi^{p}\right)$. This implies that $\mathcal{C}=1$.

LEMMA 11. Under the setting and assumptions of Lemma 10, we have $\mathrm{Cl}_{K}(\pi)=\{0\}$.

Proof. We have a natural surjection $\varphi: \mathrm{Cl}_{K}\left(\pi^{p}\right) \rightarrow \mathrm{Cl}_{K}(\pi)$ compatible with the action of $\Delta_{F}$. Let $\mathcal{C}$ be a nontrivial class in $\mathrm{Cl}_{K}\left(\pi^{p}\right)$. By Lemma 10 , the order of $\mathcal{C}$ equals $p^{e}$ for some $e \geq 1$. It suffices to show that $\varphi(\mathcal{C})=1$.

Let us first show that $\varphi\left(\mathcal{C C}^{\prime}\right)=1$. Let $\mathfrak{P}, \mathfrak{Q} \in \mathcal{C}$ be prime ideals of $K$ with $(\mathfrak{P}, \mathfrak{Q})=\left(\mathfrak{P}, \mathfrak{Q}^{\prime}\right)=1$. Then $\mathfrak{P Q}^{p^{e}-1}=a \mathcal{O}_{K}$ for some integer $a$ with $a \equiv 1 \bmod \pi^{p}$. Let $b=a\left(a^{\prime}\right)^{p-1}$ and $L=K\left(b^{1 / p}\right)$. We have

$$
b \mathcal{O}_{K}=\left(\mathfrak{P Q}^{\prime}\right)\left(\mathfrak{P}^{\prime} \mathfrak{Q}\right)^{p-1} \mathfrak{A}^{p}
$$

with

$$
\mathfrak{A}=\mathfrak{Q}^{p^{e-1}-1}\left(\mathfrak{Q}^{\prime}\right)^{p^{e}-p^{e-1}-1} .
$$

In particular, the cyclic extension $L / K$ is of degree $p$. As $b \equiv 1 \bmod \pi^{p}$, it is tame. As $b b^{\prime} \in\left(K^{\times}\right)^{p}$, there exists a tame cyclic extension $N / F$ of degree $p$ with $L=N K$. As $F$ satisfies $\left(\mathrm{A}_{p}\right), L / K$ has a NIB. Then it follows from (11) and Lemma 6 that $\varphi\left(\mathcal{C C}^{\prime}\right)=\left[\mathfrak{P Q}^{\prime}\right]_{\pi}=1$.

Let us deal with the case $e=1$. By (12), we have $\mathfrak{A}=\left(\mathfrak{Q}^{\prime}\right)^{p-2}$. By (11) and Lemma 6, the class $\varphi\left(\mathcal{C}^{\prime}\right)^{p-2}=[\mathfrak{A}]_{\pi}$ is trivial in $\mathrm{Cl}_{K}(\pi)$. This implies that $\varphi(\mathcal{C})=1$ since $\mathrm{Cl}_{K}(\pi)$ is a $p$-abelian group by Lemma 10 .

Finally, we deal with the case $e \geq 2$. Let $\mathfrak{R}, \mathfrak{Q}_{1}, \ldots, \mathfrak{Q}_{p-1} \in \mathcal{C}$ be prime ideals of $K$ which are relatively prime to each other and to their conjugates over $F$. Then

$$
\mathfrak{R}\left(\mathfrak{Q}_{1} \ldots \mathfrak{Q}_{p-1}\right)^{\left(p^{e}-1\right) /(p-1)}=a_{1} \mathcal{O}_{K}
$$

for some $a_{1} \in \mathcal{O}_{K}$ with $a_{1} \equiv 1 \bmod \pi^{p}$. Let $b_{1}=a_{1}\left(a_{1}^{\prime}\right)^{p-1}$ and $L_{1}=$ $K\left(b_{1}^{1 / p}\right)$. Then $L_{1} / K$ is of degree $p$, and has a NIB as $F$ satisfies $\left(\mathrm{A}_{p}\right)$. We have

$$
b_{1} \mathcal{O}_{K}=\left(\mathfrak{R} \mathfrak{Q}_{1} \ldots \mathfrak{Q}_{p-1}\right)\left(\mathfrak{R}^{\prime} \mathfrak{Q}_{1}^{\prime} \ldots \mathfrak{Q}_{p-1}^{\prime}\right)^{p-1} \mathfrak{B}^{p}
$$

with

$$
\mathfrak{B}=\left(\mathfrak{Q}_{1} \ldots \mathfrak{Q}_{p-1}\right)^{\left(p^{e-1}-1\right) /(p-1)}\left(\mathfrak{Q}_{1}^{\prime} \ldots \mathfrak{Q}_{p-1}^{\prime}\right)^{p^{e-1}-1} .
$$


As $L_{1} / K$ has a NIB, it follows from Lemma 6 that

$$
[\mathfrak{B}]_{\pi}=\varphi(\mathcal{C})^{p^{e-1}-1} \varphi\left(\mathcal{C}^{\prime}\right)^{(p-1)\left(p^{e-1}-1\right)}=1 .
$$

On the other hand, we have seen above that $\varphi(\mathcal{C})=\varphi\left(\mathcal{C}^{\prime}\right)^{-1}$. Hence, $\varphi\left(\mathcal{C}^{\prime}\right)^{(p-2)\left(p^{e-1}-1\right)}=1$. Therefore, $\varphi(\mathcal{C})=1$ as $\mathrm{Cl}_{K}(\pi)$ is a $p$-abelian group.

LEMMA 12. Under the setting and assumptions of Lemma 10, we have $\mathrm{Cl}_{K}(p)^{\Delta_{F}}=\{0\}$.

Proof. By Lemma 10, $\left(\mathcal{O}_{K} / p\right)^{\times} /\left[E_{K}\right]_{p}$ is a $p$-abelian group. Therefore, we see from Theorem 6 that $\left(\mathcal{O}_{F} / p\right)^{\times} \subseteq\left[E_{K}\right]_{p}$. Then we obtain $\mathrm{Cl}_{K}(p)^{\Delta_{F}}=$ $\{0\}$ from Lemmas $7($ II) and 11.

Now, Theorem 1 follows from Lemmas 11 and 12.

\section{Proofs of Theorems 3, 4 and Proposition}

5.1. Proof of Theorem 4. In all what follows, we let $p=3$ and $\pi=\pi_{3}=$ $\zeta_{3}-1$. We begin with the following lemma.

Lemma 13. Let $F, K$ be as in Theorem $4, N / F$ a tame cyclic cubic extension, and $L=N K$. Then $L / K$ has a NIB if and only if there exists an integer $\lambda \in \mathcal{O}_{K}$ with $L=K\left(\left(\lambda\left(\lambda^{\prime}\right)^{2}\right)^{1 / 3}\right)$ satisfying the following conditions:

(i) $\lambda \mathcal{O}_{K}$ is square free and $\left(\lambda, \lambda^{\prime}\right)=1$,

(ii) $\varepsilon_{1}^{3} \lambda\left(\lambda^{\prime}\right)^{2} \equiv 1 \bmod \pi^{3}$ for some unit $\varepsilon_{1} \in E_{K}$,

(iii) $\lambda \equiv \varepsilon_{2} \bmod 3$ for some unit $\varepsilon_{2} \in E_{K}$.

Proof. We can easily show the "if" part using Theorem 5 by an argument similar to the proof of Lemma 8 . So, we assume that $L / K$ has a NIB, and show the "only if" part. As $L / K$ has a NIB, there exists an integer $a \in \mathcal{O}_{K}$ relatively prime to 3 with $L=K\left(a^{1 / 3}\right)$ satisfying the conditions in Theorem 5 . As $a \mathcal{O}_{K}$ is cube free, $a \mathcal{O}_{K}=\mathfrak{A}_{1} \mathfrak{A}_{2}^{2}$ for some square free integral ideals $\mathfrak{A}_{i}$ of $K$ with $\left(\mathfrak{A}_{1}, \mathfrak{A}_{2}\right)=1$. By Lemma 6 , the ideals $\mathfrak{A}_{1}$ and $\mathfrak{A}_{2}$ are principal. As $L=N K$, we must have $a a^{\prime} \in\left(K^{\times}\right)^{3}$. From this, it follows that $\mathfrak{A}_{2}=\mathfrak{A}_{1}^{\prime}$. Hence, we can write $a=\eta \lambda\left(\lambda^{\prime}\right)^{2}$. Here, $\eta$ is a unit of $K$, and $\lambda \in \mathcal{O}_{K}$ is an integer such that $\lambda \mathcal{O}_{K}$ is square free and $\left(\lambda, \lambda^{\prime}\right)=1$. From $a a^{\prime} \in\left(K^{\times}\right)^{3}$, we have $\eta \eta^{\prime}=\eta_{1}^{3}$ for some $\eta_{1} \in E_{K}$. As $[K: F]=2$, we see that $\eta_{1} \in E_{F}$. Further, as the quotient $E_{F} / N_{K / F} E_{K}$ is of exponent 2, we have $\eta_{1}=\eta_{2} \eta_{2}^{\prime}$ for some $\eta_{2} \in E_{K}$. Therefore, $\eta=\eta_{2}^{3} \delta$ for some unit $\delta \in E_{K}$ with $\delta \delta^{\prime}=1$. Hence, replacing $a$ with $a / \eta_{2}^{3}$ and $\lambda$ with $\delta^{\prime} \lambda$, we can write $a=\lambda\left(\lambda^{\prime}\right)^{2}$. Now, the assertion follows from Lemma 5 .

We now turn to the proof of Theorem 4. It suffices to show the "if" part. Assume that $L / K$ has a NIB, and choose $\lambda, \varepsilon_{1}, \varepsilon_{2}$ as in Lemma 13 . 
Let $b=\varepsilon_{1}^{3} \lambda\left(\lambda^{\prime}\right)^{2}$, and $\beta=b^{1 / 3}(\equiv 1 \bmod \pi)$. We have $\beta^{\prime}=\varepsilon_{1} \varepsilon_{1}^{\prime} \lambda \lambda^{\prime} / \beta$. By Lemma 1,

$$
\mathcal{O}_{L}^{(1)}=\mathcal{O}_{K} \beta, \quad \mathcal{O}_{L}^{(2)}=\mathcal{O}_{K} \frac{\varepsilon_{1} \varepsilon_{1}^{\prime} \lambda \lambda^{\prime}}{\beta} .
$$

By Lemma 3, it suffices to show that there exists a unit $\eta \in E_{K}$ such that

$$
1+\beta \eta+\frac{\varepsilon_{1} \varepsilon_{1}^{\prime} \lambda \lambda^{\prime}}{\beta} \eta^{\prime} \equiv 0 \bmod 3 .
$$

This is equivalent to saying that

$$
(\beta \eta)^{2}+\beta \eta+\varepsilon_{1} \varepsilon_{1}^{\prime} \lambda \lambda^{\prime} \eta \eta^{\prime} \equiv 0 \bmod 3 .
$$

As $\beta \equiv 1 \bmod \pi$, we see that $\beta^{\prime} \equiv 1 \bmod \pi$ and hence

$$
\varepsilon_{1} \varepsilon_{1}^{\prime} \lambda \lambda^{\prime} \equiv 1 \bmod \pi
$$

On the other hand, we have

$$
b / b^{\prime} \equiv \varepsilon_{1}^{3}\left(\varepsilon_{1}^{\prime}\right)^{-3} \lambda^{-1} \lambda^{\prime} \equiv 1 \bmod \pi .
$$

From (14) and (15), it follows that $\varepsilon_{1}^{4}\left(\varepsilon_{1}^{\prime}\right)^{-2}\left(\lambda^{\prime}\right)^{2} \equiv 1 \bmod \pi$, and hence

$$
\lambda^{2} \varepsilon_{1}^{-2}\left(\varepsilon_{1}^{\prime}\right)^{4}-1=\left(\lambda \varepsilon_{1}^{-1}\left(\varepsilon_{1}^{\prime}\right)^{2}-1\right)\left(\lambda \varepsilon_{1}^{-1}\left(\varepsilon_{1}^{\prime}\right)^{2}+1\right) \equiv 0 \bmod \pi .
$$

CLAIM. $\lambda \varepsilon_{1}^{-1}\left(\varepsilon_{1}^{\prime}\right)^{2} \equiv 1 \bmod \pi$.

Indeed, let $\mathfrak{P}$ be a prime ideal of $K$ over $p$, and $e=\operatorname{ord}_{\mathfrak{P}} \pi$. From the above congruence, we see that $\lambda \varepsilon_{1}^{-1}\left(\varepsilon_{2}^{\prime}\right)^{2} \equiv 1$ or $-1 \bmod \mathfrak{P}^{e}$ because

$$
\left(\lambda \varepsilon_{1}^{-1}\left(\varepsilon_{2}^{\prime}\right)^{2}-1, \lambda \varepsilon_{1}^{-1}\left(\varepsilon_{2}^{\prime}\right)^{2}+1\right) \mid 2 \text { and } p \neq 2 .
$$

Assume that $x=\lambda \varepsilon_{1}^{-1}\left(\varepsilon_{1}^{\prime}\right)^{2} \equiv-1 \bmod \mathfrak{P}^{e}$. By the above, we have $x \equiv$ $\pm 1 \bmod \left(\mathfrak{P}^{\prime}\right)^{e}$. Hence, $\left(x^{\prime}\right)^{2} \equiv 1 \bmod \mathfrak{P}^{e}$. Thus, $b=x\left(x^{\prime}\right)^{2} \equiv-1 \bmod \mathfrak{P}^{e}$. This contradicts $b \equiv 1 \bmod \pi$. Hence, the claim is shown.

Let $\eta=\left(\varepsilon_{2} \varepsilon_{1}^{-1}\left(\varepsilon_{1}^{\prime}\right)^{2}\right)^{-1}$. Then, by the Claim and Lemma 13(iii), we see that

$$
\eta \equiv 1 \bmod \pi \quad \text { and } \quad \varepsilon_{1} \varepsilon_{1}^{\prime} \lambda \lambda^{\prime} \eta \eta^{\prime} \equiv 1 \bmod 3 .
$$

Therefore, as $\beta \equiv 1 \bmod \pi$, congruence (13) holds. Hence, $N / F$ has a NIB.

5.2. Proof of Theorem 3. By Theorem 1, it suffices to show the "if" part. Assume that $F$ satisfies $\mathrm{Cl}_{K}(\pi)=\{0\}$ and $\mathrm{Cl}_{K}(3)^{\Delta_{F}}=\{0\}$. In particular, $h_{K}=1$. Let $N / F$ be an arbitrary tame cyclic cubic extension, and $L=N K$. By Theorem 4 , it suffices to show that $L / K$ has a NIB. As $h_{K}=1$, we can write $L=K\left(a^{1 / 3}\right)$ with

$$
a=\varepsilon \prod_{i=1}^{r}\left(\pi_{i}^{e_{i}}\left(\pi_{i}^{\prime}\right)^{f_{i}}\right) \prod_{j=1}^{s} \varrho_{j}^{g_{j}} \quad\left(e_{i} \in\{1,2\}, f_{i}, g_{j} \in\{0,1,2\}\right) .
$$

Here, $\varepsilon \in E_{K}$, and $\pi_{i}$ (resp. $\varrho_{j}$ ) are integers of $K$ relatively prime to 3 such that $\pi_{i} \mathcal{O}_{K}$ (resp. $\left.\varrho_{j} \mathcal{O}_{K}\right)$ are prime ideals of $K$ of relative degree one (resp. two) over $F$. The integers $\pi_{i}, \varrho_{j}$ are chosen so that $N_{K / F} \pi_{i}$ and $\varrho_{j}$ are 
relatively prime to each other. As $L=N K$, we have $a a^{\prime} \in\left(K^{\times}\right)^{3}$. Therefore, it follows that $e_{i}+f_{i}=3$ and $g_{j}=0$. It also follows that $\varepsilon=\varepsilon_{1}^{3} \delta$ for some units $\varepsilon_{1}, \delta \in E_{K}$ with $\delta \delta^{\prime}=1$. Now, letting

$$
b=\delta^{\prime} \prod_{e_{i}=1} \pi_{i} \prod_{e_{i}=2} \pi_{i}^{\prime},
$$

we have $a=\varepsilon_{1}^{3} b\left(b^{\prime}\right)^{2}$. Here, in the first (resp. second) product, $i$ runs over integers $1 \leq i \leq r$ with $e_{i}=1$ (resp. $e_{i}=2$ ). As $b \mathcal{O}_{K}$ is square free and $\left(b, b^{\prime}\right)=1$, we see that $L / K$ has a NIB by Lemma 8 .

5.3. Proof of Proposition. It is known and easy to show that $F=$ $\mathbb{Q}(\sqrt{-3})$ satisfies $\left(\mathrm{A}_{3}\right)$ (cf. $[4$, p. 110$\left.]\right)$. Let $K=\mathbb{Q}(\sqrt{\ell}, \sqrt{-3})$ be a $(2,2)$ extension of $\mathbb{Q}$ with $\ell$ a square free integer, and let $Q_{K}(=1,2)$ be the unit index of $K$, and $\varepsilon$ a fundamental unit of $K$. Let $\varepsilon_{0}>0$ be a fundamental unit of the maximal real subfield $K^{+}$of $K$. We can calculate $Q_{K}$ and $\varepsilon$ using a classical result in Hasse [7, Section 26]. When $Q_{K}=1$, we have $\varepsilon=\varepsilon_{0}$. When $Q_{K}=2$, we can choose $\varepsilon$ so that

$$
\varepsilon^{2}=\sqrt{-1} \cdot \varepsilon_{0} \quad \text { or } \quad-\varepsilon_{0}
$$

according to whether $\sqrt{-1} \in K^{\times}$or not. By Uchida [14], there are exactly $13 K$ 's with $h_{K}=1$, namely,

(a) $\ell=2,5,17,41,89$,

(b) $\ell=-1,-2,-11$, or

(c) $\ell=-7,-19,-43,-67,-163$.

For this, see also Yamamura [16]. By Theorem 3, a quadratic field $F$ satisfying $\left(\mathrm{A}_{3}\right)$ is contained in these $K$. For a finite abelian group $A$, we write $A=\left(n_{1}, \ldots, n_{r}\right)$ when $A$ is isomorphic to the additive group $\mathbb{Z} / n_{1} \oplus$ $\ldots \oplus \mathbb{Z} / n_{r}$.

First, let us deal with quadratic fields contained in those $K$ in (c). We see that $Q_{K}=2$ and $\left(\mathcal{O}_{K} / \pi\right)^{\times}=(8)$. We have $\varepsilon_{0} \equiv \pm 1 \bmod \pi$ as 3 is ramified in $K^{+}$. Hence, by (16), the order of the class $[\varepsilon]_{\pi}$ divides 4 . Therefore, we see that $\left[E_{K}\right]_{\pi} \varsubsetneqq\left(\mathcal{O}_{K} / \pi\right)^{\times}$and $\mathrm{Cl}_{K}(\pi) \neq\{0\}$. Hence, by Theorem 3 , any quadratic field $F \neq \mathbb{Q}(\sqrt{-3})$ contained in these $K$ does not satisfy $\left(\mathrm{A}_{3}\right)$.

Next, let us deal with those $K$ in (b). For these $K$, we see that $Q_{K}=2$, and that by (16),

$$
\varepsilon=(-1-\sqrt{-1}+\sqrt{-3}-\sqrt{3}) / 2, \sqrt{-2}+\sqrt{-3}, \sqrt{-11}+2 \sqrt{-3},
$$

respectively. Using this, we easily see that $\left(\mathcal{O}_{K} / 3\right)^{\times}=\left[E_{K}\right]_{3}$. As $h_{K}=1$, this implies that $\mathrm{Cl}_{K}(3)=\{0\}$. Then it follows from [8, V, Proposition 2] that $K$ satisfies $\left(\mathrm{A}_{3}\right)$. Therefore, by Theorem 4 , all quadratic fields contained in these $K$ satisfy $\left(\mathrm{A}_{3}\right)$.

Finally, let us deal with those $K$ in (a). For these $K$, we have $Q_{K}=1$ and $\left(\mathcal{O}_{K} / \pi\right)^{\times}=(8)$. Using $\varepsilon=\varepsilon_{0} \equiv \pm 1 \pm \sqrt{\ell} \bmod 3$ and $\ell \equiv-1 \bmod 3$, we 
see that the order of the class $[\varepsilon]_{\pi}$ (resp. $\left.[\varepsilon]_{3}\right)$ equals 8 . Hence, $\left(\mathcal{O}_{K} / \pi\right)^{\times}=$ $\left[E_{K}\right]_{\pi}$. This implies that $\mathrm{Cl}_{K}(\pi)=\{0\}$ as $h_{K}=1$. Let $F=\mathbb{Q}(\sqrt{\ell})$ be a real quadratic field contained in these $K$. We have $\left(\mathcal{O}_{F} / 3\right)^{\times}=(8)$ and $\left(\mathcal{O}_{K} / 3\right)^{\times}=(3,3,8)$. Thus $\left(\mathcal{O}_{F} / 3\right)^{\times} \subseteq\left[E_{K}\right]_{3}$ since the class $[\varepsilon]_{3}$ is of order 8 . By Lemma 7, this implies that $\mathrm{Cl}_{K}(3)^{\Delta_{F}}=\{0\}$. Hence, by Theorem 3, a real quadratic field $F=\mathbb{Q}(\sqrt{\ell})$ with $\ell$ in (a) satisfies $\left(\mathrm{A}_{3}\right)$. Let $F=\mathbb{Q}(\sqrt{-3 \ell})$ be an imaginary quadratic field contained in these $K$. We see that $\left(\mathcal{O}_{F} / 3\right)^{\times}=$ $(2,3)$, and that $\left[E_{K}\right]_{3}=(3,8)$ is generated by the classes $\left[\zeta_{3}\right]_{3}$ and $[\varepsilon]_{3}$. Let $x=1+\sqrt{-3 \ell}$. We see that the class $[x]_{3} \in\left(\mathcal{O}_{F} / 3\right)^{\times}$is of order 3 but $x \not \equiv \zeta_{3}, \zeta_{3}^{2} \bmod 3$. Hence, $\left(\mathcal{O}_{F} / 3\right)^{\times} \varsubsetneqq\left[E_{K}\right]_{3}$. Therefore, any imaginary quadratic field $F \neq \mathbb{Q}(\sqrt{-3})$ contained in these $K$ does not satisfy $\left(\mathrm{A}_{3}\right)$. Thus, we have shown the Proposition.

Acknowledgements. The author was partially supported by Grant-inAid for Scientific Research (C) (no. 13640036), the Ministry of Education, Culture, Sports, Science and Technology of Japan.

Note added in proof. After this paper was accepted for publication, a paper of J. E. Carter appeared in Arch. Math. (Basel) 81 (2003), 266-271. He gives a necessary and sufficient condition for a number field $F$ to satisfy $\left(\mathrm{A}_{3}\right)$. His condition is different from ours, and is obtained by a different method. As an application, he determines the quadratic fields satisfying $\left(A_{3}\right)$. However, some quadratic fields seem to be neglected in his result.

\section{References}

[1] J. Brinkhuis, Normal integral bases and Spiegelungssatz of Scholz, Acta Arith. 69 (1995), 1-9.

[2] R. J. Chapman, Kummer theory and Galois module structure in global function fields, Math. Z. 208 (1991), 250-260.

[3] A. Fröhlich and M. J. Taylor, Algebraic Number Theory, Cambridge Univ. Press, Cambridge, 1991.

[4] E. J. Gómez Ayala, Bases normales d'entiers dans les extensions de Kummer de degré premier, J. Théor. Nombres Bordeaux 6 (1994), 95-116.

[5] C. Greither, On normal integral bases in ray class fields over imaginary quadratic fields, Acta Arith. 78 (1997), 315-329.

[6] C. Greither, D. Replogle, K. Rubin and A. Srivastav, Swan modules and HilbertSpeiser number fields, J. Number Theory 79 (1999), 164-173.

[7] H. Hasse, Über die Klassenzahl abelscher Zahlkörper, Akademie Verlag, Berlin, 1952.

[8] H. Ichimura, Note on the ring of integers of a Kummer extension of prime degree, $I V$, Proc. Japan Acad. Ser. A Math. Sci. 77 (2001), 92-94; V, ibid. 78 (2002), 76-79.

[9] -, On a theorem of Childs on normal bases of rings of integers, J. London Math. Soc. (2) 68 (2003), 25-36; addendum, ibid., in press.

[10] - On the ring of integers of a tame Kummer extension over a number field, J. Pure Appl. Algebra 187 (2004), 169-182.

[11] - On a theorem of Kawamoto on normal bases of rings of integers, Tokyo J. Math., in press. 
[12] F. Kawamoto, On normal integral bases, Tokyo J. Math. 7 (1984), 221-231.

[13] —, Remark on "On normal integral bases", ibid. 8 (1985), 275.

[14] K. Uchida, Imaginary abelian number fields of degrees $2^{m}$ with class number one, in: Proc. Internat. Conf. on Class Numbers and Fundamental Units of Algebraic Number Fields (Katata, 1986), Y. Yamamoto and H. Yokoi (eds.), Nagoya Univ., Nagoya, 1986, 151-170.

[15] L. C. Washington, Introduction to Cyclotomic Fields, 2nd ed., Springer, Berlin, 1997.

[16] K. Yamamura, The determination of imaginary abelian fields of class number one, Math. Comp. 62 (1994), 899-921.

Department of Mathematics

Yokohama City University

22-2, Seto, Kanazawa-ku

Yokohama 236-0027, Japan

E-mail: ichimura@yokohama-cu.ac.jp

Received on 19.8.2003

and in revised form on 9.10.2003 